\title{
Automatic Theorem-Proving in Combinatorics on Words
}

\author{
Dane Henshall and Jeffrey Shallit \\ School of Computer Science, University of Waterloo, Waterloo, ON N2L 3G1 Canada \\ dhenshall@uwaterloo.ca, shallit@cs.uwaterloo.ca
}

\begin{abstract}
We describe a technique for mechanically proving certain kinds of theorems in combinatorics on words, using automata and a package for manipulating them. We illustrate our technique by solving, purely mechanically, an open problem of Currie and Saari on the lengths of unbordered factors in the Thue-Morse sequence.
\end{abstract}

Dedicated to the memory of Sheng Yu (1950-2012): friend and colleague

\section{Introduction}

The title of this paper is a bit of a pun. On the one hand, we are concerned with certain natural questions about automatic sequences: sequences over a finite alphabet where the $n$ 'th term is expressible as a finite-state function of the base$k$ representation of $n$. On the other hand, we are interested in answering these questions purely mechanically, in an automated fashion.

Let $\mathbf{x}=(a(n))_{n \geq 0}$ be an infinite sequence over a finite alphabet $\Delta$. Then $\mathbf{x}$ is said to be $k$-automatic if there is a deterministic finite automaton $M$ taking as input the base- $k$ representation of $n$, and having $a(n)$ as the output associated with the last state encountered [3]. In this case, we say that $M$ generates the sequence $\mathbf{x}$.

For example, in Figure 1, we give an automaton generating the well-known Thue-Morse sequence $\mathbf{t}=t(0) t(1) t(2) \cdots=011010011001 \cdots$ [2]. The input is $n$, expressed in base 2 , and the output is the number contained in the state last reached. Thus $t(n)$ is the sum, modulo 2 , of the binary digits of $n$.

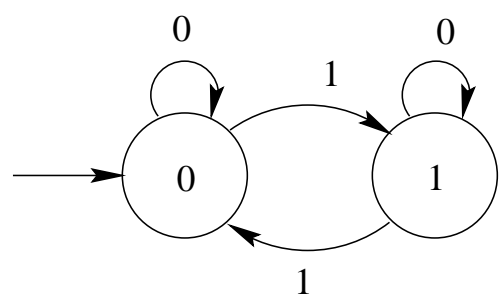

Fig. 1. A finite automaton generating the Thue-Morse sequence 
For at least 25 years, researchers have been interested in the algorithmic decidability of assertions about automatic sequences. For example, in one of the earliest results, Honkala [17] showed that, given an automaton, it is decidable if the sequence it generates is ultimately periodic.

Recently, Allouche et al. [1] found a different proof of Honkala's result using a more general technique. Using this technique, they were able to give algorithmic solutions to many classical problems from combinatorics on words such as

Given an automaton, is the generated sequence squarefree? Or overlapfree?

We write $\mathbf{x}[i]=a(i)$, and we let $\mathbf{x}[i . . i+n-1]$ denote the factor of length $n$ beginning at position $i$ in $\mathbf{x}$. A sequence is said to be squarefree if it contains no factor of the form $x x$, where $x$ is a nonempty word, and is said to overlapfree if it contains no factor of the form ayaya, where $a$ is a single letter and $y$ is a possibly empty word.

The technique of Allouche et al. is at its core, very similar to work of Büchi, Bruyère, Michaux, Villemaire, and others, involving formal logic; see, e.g., [5]. The basic idea is as follows: given the automaton $M$, and some predicate $P(n)$ we want to check, we alter $M$ by a series of transformations to a new automaton $M^{\prime}$ that accepts the base- $k$ representations of those integers $n$ for which $P(n)$ is true. Then we can check the assertion " $\exists n P(n)$ " simply by checking if $M^{\prime}$ accepts anything (which can be done by a standard depth-first search on the underlying directed graph of the automaton). We can check the assertion " $\forall n P(n)$ " by checking if $M^{\prime}$ accepts everything. And we can check assertions like " $P(n)$ holds for infinitely many $n$ " by checking if $M^{\prime}$ has a reachable cycle from which a final state is reachable.

Using this idea, Allouche et al. were able to show to reprove, purely mechanically using a computer program, the classic theorem of Thue 24|25|4 that the Thue-Morse sequence $\mathbf{t}$ is overlapfree.

More recently, the technique has been applied to give decision procedures for other properties of automatic sequences. For example, Charlier et al. [6] showed that it can be used to decide if a given $k$-automatic sequence

- contains powers of arbitrarily large exponent;

- is recurrent;

- is uniformly recurrent.

A sequence is said to be recurrent if every factor that occurs, occurs infinitely often. A sequence $\mathbf{x}$ is said to be uniformly recurrent if it is recurrent and furthermore for each finite factor $w$ occurring in $\mathbf{x}$, there is a constant $c(w)$ such that two consecutive occurrences of $w$ are separated by at most $c(w)$ positions.

More recently, variations of the technique have been used to

- compute the critical exponent;

- compute the initial critical exponent;

- decide if a sequence is linearly recurrent;

- compute the Diophantine exponent.

(For definitions of these terms see 22.) 


\section{The decision procedure}

In [6] we have the following theorem:

Theorem 1. If we can express a property of a $k$-automatic sequence $\mathbf{x}$ using quantifiers, logical operations, integer variables, the operations of addition, subtraction, indexing into $\mathbf{x}$, and comparison of integers or elements of $\mathbf{x}$, then this property is algorithmically decidable.

Let us outline how the decision procedure works.

First, the input to the decision procedure: an automaton $M=\left(Q, \Sigma_{k}, \Delta, \delta, q_{0}, \tau\right)$ generating the $k$-automatic sequence $\mathbf{x}$. Here

- $Q$ is a nonempty set of states;

$-\Sigma_{k}:=\{0,1, \ldots, k-1\}$

$-\Delta$ is the output alphabet;

$-\delta: Q \times \Sigma \rightarrow Q$ is the transition function;

$-q_{0}$ is the initial state; and

$-\tau: Q \rightarrow \Delta$ is the output mapping.

In this paper, we assume that the automaton takes as input the representation of $n$ in base $k$, starting with the least significant digit; we call this the reversed representation of $n$ and write it as $(n)_{k}$. We allow leading zeroes in the representation (which, because of our convention, are actually trailing zeroes). Thus, for example, 011 and 01100 are both acceptable representations for 6 in base 2 .

We might also need to encode pairs, triples, or $r$-tuples of integers. We handle these by first padding the reversed representation of the smaller integer with trailing zeroes, and then coding the $r$-tuple as a word over $\Sigma_{k}^{r}$. For example, the pair $(20,13)$ could be represented in base- 2 as

$$
[0,1][0,0][1,1][0,1][1,0],
$$

where the first components spell out 00101 and the second components spell out 10110. Of course, there are other possible representations, such as

$$
[0,1][0,0][1,1][0,1][1,0][0,0],
$$

which correspond to non-canonical representations having trailing zeroes; these are also permitted.

Rather than present a detailed proof, we illustrate the idea of the decision procedure in the proof of the following new result:

Theorem 2. The following problem is algorithmically decidable: given two $k$ automatic sequences $\mathbf{x}$ and $\mathbf{y}$, generated by automata $M_{1}$ and $M_{2}$, respectively, decide if $\mathbf{x}$ is a shift of $\mathbf{y}$ (that is, decide if there exists a constant $c$ such that $\mathbf{x}[n]=\mathbf{y}[n+c]$ for all $n \geq 0$. 
Proof. We first create an NFA $M$ that accepts the language

$$
\left\{(c)_{k}: \exists n \text { such that } \mathbf{x}[n] \neq \mathbf{y}[n+c]\right\} .
$$

To do so, on input $(c)_{k}, M$

- guesses $w_{1}=(n)_{k}$ nondeterministically (perhaps with trailing zeroes appended),

- simulates $M_{1}$ on $w_{1}$,

- adds $n$ to $c$ and computes the base- $k$ representation of $w_{2}=(n+c)_{k}$ digitby-digit "on the fly", keeping track of carries, as necessary, and simulates $M_{2}$ on $w_{2}$, and

- accepts if the outputs of both machine differ.

We now convert $M$ to a DFA $M^{\prime}$, and change final states to non-final (and vice versa). Then $M^{\prime}$ accepts the language

$$
\left\{(c)_{k}: \mathbf{x}[n]=\mathbf{y}[n+c] \text { for all } n \geq 0\right\} .
$$

Thus, $\mathbf{x}$ is a shift of $\mathbf{y}$ if and only if $M^{\prime}$ accepts any word, which is easily checked through depth-first search.

Remark 1. As we can see, the size of the automata involved depends, in an unpleasant way, on the number of quantifiers needed to state the logical expression characterizing the property being checked, because existential quantifiers are implemented through nondeterminism, and universal quantifiers are implemented through nondeterminism and complementation (which is implemented in a DFA by exchange of the role final and non-final states). Thus each new quantifier could increase the current number of states, say $n$, to $2^{n}$ using the subset construction. If the original automata have at most $N$ states, it follows that the running time is bounded by an expression of the form

$$
2^{2^{\cdot} \cdot 2^{p(N)}}
$$

where $p$ is a polynomial and the number of exponents in the tower is one less than the number of quantifiers in the logical formula characterizing the property being checked.

This extraordinary computational complexity raises the natural question of whether the decision procedure could actually be implemented for anything but toy examples. Luckily the answer seems to be yes - at least in some cases as we will see below.

\section{Borders}

A word $w$ is bordered if it begins and ends with the same word $x$ with $0<$ $|x| \leq|w| / 2$; Otherwise it is unbordered. An example in English of a bordered 
word is entanglement. A bordered word is also called bifix in the literature, and unbordered words are also called bifix-free or primary.

Bordered and unbordered words have been actively studied in the literature, particularly with regard to the Ehrenfeucht-Silberger problem; see, for example, [13]18]10|11|14 $15|7| 16[19] 12$, just to name a few.

Currie and Saari 8 s studied the unbordered factors of the Thue-Morse sequence $\mathbf{t}$. They proved that if $n \not \equiv 1(\bmod 6)$, then $\mathbf{t}$ has an unbordered factor of length $n$. (Also see [21, Lemma 4.10 and Problem 4.1].) However, this is not a necessary condition, as

$$
\mathbf{t}[39 . .69]=0011010010110100110010110100101,
$$

which is an unbordered factor of length 31. Currie and Saari left it as an open problem to give a complete characterization of the integers $n$ for which $\mathbf{t}$ has an unbordered factor of length $n$.

The following theorem and proof, quoted practically verbatim from [ $[\underline{6}$, shows that, more generally, the characteristic sequence of $n$ for which a given $k$ automatic sequence has an unbordered factor of length $n$, is itself $k$-automatic:

Theorem 3. Let $\mathbf{x}=a(0) a(1) a(2) \cdots$ be a k-automatic sequence. Then the associated infinite sequence $\mathbf{b}=b(0) b(1) b(2) \cdots$ defined by

$$
b(n)= \begin{cases}1, & \text { if } \mathbf{x} \text { has an unbordered factor of length } n ; \\ 0, & \text { otherwise; }\end{cases}
$$

is k-automatic.

Proof. The sequence $\mathbf{x}$ has an unbordered factor of length $n$

iff

$\exists j \geq 0$ such that the factor of length $n$ beginning at position $j$ of $\mathbf{x}$ is unbordered iff

there exists an integer $j \geq 0$ such that for all possible lengths $l$ with $1 \leq l \leq n / 2$, there is an integer $i$ with $0 \leq i<l$ such that the supposed border of length $l$ beginning and ending the factor of length $n$ beginning at position $j$ of $\mathbf{x}$ actually differs in the $i$ 'th position

iff

there exists an integer $j \geq 0$ such that for all integers $l$ with $1 \leq l \leq n / 2$ there exists an integer $i$ with $0 \leq i<l$ such that $\mathbf{x}[j+i] \neq \mathbf{x}[j+n-l+i]$.

Now assume $\mathbf{x}$ is a $k$-automatic sequence, generated by some finite automaton. We show how to implement the characterization given above with an automaton.

We first create an NFA that given the $(j, l, n)_{k}$ guesses the base- $k$ representation of $i$, digit-by-digit, checks that $i<l$, computes $j+i$ and $j+n-l+i$ on the fly, and checks that $\mathbf{x}[j+i] \neq \mathbf{x}[j+n-l+i]$. If such an $i$ is found, it accepts. We then convert this to a DFA, and interchange accepting and nonaccepting 
states. This DFA $M_{1}$ accepts $(j, l, n)_{k}$ such that there is no $i, 0 \leq i<l$ such that $\mathbf{x}[j+i]=\mathbf{x}[j+n-l+i]$. We then use $M_{1}$ as a subroutine to build an NFA $M_{2}$ that on input $(j, n)_{k}$ guesses $l$, checks that $1 \leq l \leq n / 2$, and calls $M_{1}$ on the result. We convert this to a DFA and interchange accepting and nonaccepting states to get $M_{3}$. Finally, this $M_{3}$ is used as a subroutine to build an NFA $M_{4}$ that on input $n$ guesses $j$ and calls $M_{3}$.

The characteristic sequence of these integers $n$ is therefore $k$-automatic.

Since the proof is constructive, one can, in principle, carry out the construction to get an explicit description of the lengths for which the Thue-Morse sequence has an unbordered factor.

Doing so results in the following theorem:

Theorem 4. There is an unbordered factor of length $n$ in $\mathbf{t}$ if and only if the base-2 representation of $n$ (starting with the most significant digit) is not of the form $1\left(01^{*} 0\right)^{*} 10^{*} 1$.

Proof. The proof of this theorem is purely mechanical, and it involves performing a sequence of operations on finite automata. The second author wrote a program in $\mathrm{C}++$, using his own automata package, to perform these operations. There are four stages to the computation, which are described in detail below.

\section{Stage 1}

Let $T$ be the automaton of Figure 1 generating the Thue-Morse sequence t. Stage 1 takes $T$ as input and outputs an automaton $M_{1}$, where $M_{1}$ accepts $w \in\left(\{0,1\}^{4}\right)^{*}$ if and only if $w$ is the base-2 representation of some $(n, j, l, i) \in S_{1}$, where

$$
S_{1}=\{(n, j, l, i): 0<l \leq n / 2 \text { and } i<j \text { and } \mathbf{t}[j+i] \neq \mathbf{t}[n+j-l+i]\} .
$$

The size of $M_{1}$ was only 102 states. However, since the input alphabet for $M_{1}$ is of size $2^{4}=16$, a considerable amount of complexity is being stored in the transition matrix. Stage 1 passed all 1.3 million tests meant to ensure that $M_{1}$ corresponds to $S_{1}$.

\section{Stage 2}

The purpose of Stage 2 is to remove the variable $i$ by simulating it. The resulting machine, after being negated, accepts $(n, j, l)$ iff the length $n$ factor of $t$ starting at index $j$ has a border of length $l$. So Stage 2 produces the automaton $M_{2}$, which is the negation of the result of simulating $i$. More formally, $M_{2}$ accepts a word $w \in\left(\{0,1\}^{3}\right)^{*}$ if and only if $w$ is the base-2 representation of some $(n, j, l) \in S_{2}$, where

$$
S_{2}=\left\{(n, j, l): \nexists i \text { for which }(n, j, l, i) \in S_{1}\right\}
$$

The size of $M_{2}$ after subset construction was 8689 states, and it minimized down to 127 states. The output of Stage 2 passed all 1.6 million tests meant to 
ensure that $M_{2}$ corresponds to $S_{2}$.

\section{Stage 3}

The purpose of Stage 3 is to remove $l$ by simulating it. By the end of Stage 3 , most of the work has already been done. The output of Stage $3, M_{3}$, accepts an input word $w \in\left(\{0,1\}^{2}\right)^{*}$ if and only if $w$ is the base-2 representation of some $(n, j) \in S_{3}$, where

$$
S_{3}=\left\{(n, j): \nexists l \text { such that }(n, j, l) \in S_{2}\right\}
$$

or, in other words

$$
S_{3}=\{(n, j): \mathbf{t} \text { has an unbordered factor of length } n \text { at index } j\} .
$$

The size of $M_{3}$ after subset construction was 1987 states, and it minimized down to 263 states. The output of Stage 3 passed all 1.9 million tests meant to ensure that $M_{3}$ corresponds to $S_{3}$.

\section{Stage 4}

Finally, Stage 4 simulates $j$ on $M_{3}$ and negates the result. So the output of Stage 3 is an automaton that accepts the binary representation of a positive integer $n>1$ if and only if the Thue-Morse word has no unbordered factor of length $n$. Formally put, the automaton $M_{4}$ produced by Stage 4 accepts a word $w \in\{0,1\}^{*}$ if and only if $w$ is the base-2 representation of some $n \in S_{4}$, where

$$
S_{4}=\left\{n \in \mathbb{N}: n>1, \nexists j \text { for which }(n, j) \in S_{3}\right\} .
$$

The size of $M_{4}$ after subset construction is 2734 states, and it minimized to 7 states. $M_{4}$ accepts the reverse of $1\left(01^{*} 0\right)^{*} 10^{*} 1$. Therefore the Thue-Morse word has an unbordered factor of length $n$ if and only if the base- 2 representation of $n$ (starting with the most significant digit) is not of the form $1\left(01^{*} 0\right)^{*} 10^{*} 1$.

The total computation took 9 seconds of CPU time on a $2.9 \mathrm{GHz}$ Dell XPS laptop.

Remark 2. Here are some additional implementation details.

In order to implement the needed operations on automata, we must decide on an encoding of elements of $\left(\Sigma_{k}^{n}\right)^{*}$. We could do this by performing a perfect shuffle of each individual word over $\Sigma_{k}^{*}$, or by letting the alphabet itself be represented by $k$-tuples. The decision represents a tradeoff between state size and alphabet size. We used the latter representation, since (a) it makes the algorithms considerably easier to implement and understand and (b) decreases the number of states needed.

It was mentioned earlier how many tests were passed in each stage. In order to make sure that the final automaton is what we expect, a number of tests are run after each stage on the output of that stage. 
For example, let $\mathbf{x}$ be an automatic sequence. The testing framework requires a $\mathrm{C}++$ function which given $n$ computes $\mathbf{x}[n]$. Before any operations are done, the automaton given for $\mathbf{x}$ is tested against the $\mathrm{C}++$ function to make sure that they match for the first 10,000 elements. Then, at each stage before Stage 4 the resulting automaton is tested to give confidence that the operations on the automata are giving the desired results.

For example, after Stage 2 of computing the set of lengths for which there exists an unbordered factor of an automatic sequence $\mathbf{x}$, we expect the machine $M_{2}$ to accept the language $S_{2}$, where

$$
S_{2}=\{(n, j, l): \nexists i \text { for which } \mathbf{x}[j+i]=\mathbf{x}[n+j-l+i]\}
$$

This is then tested by making sure $M_{2}$ accepts $(n, j, l)_{k}$ if and only if $(n, j, l) \in S_{2}$ for all $n, j, l \leq 1400$. These tests were invaluable to debugging, and provide confidence in the final result of the computation.

Finally, we have to address the issue of multiple representations. It is easy to forget that automata accept words in $\Sigma_{k}{ }^{*}$, and not integers. For some operations, such as complement and intersection, it is crucial that if one binary representation is accepted by the automaton, then all binary representations must be accepted.

\section{Additional results}

We also applied our decision procedure above to two other famous sequences: the Rudin-Shapiro sequence 20]23] and the paperfolding sequence [9].

For a word $w \in 1(0+1)^{*}$, we define $a_{w}(n)$ to be the number of (possibly overlapping) occurrences of $w$ in the (ordinary, unreversed) base- 2 representation of $n$. Thus, for example, $a_{11}(7)=2$.

The Rudin-Shapiro sequence $\mathbf{r}=r(0) r(1) r(2) \cdots$ is then defined to be $r(n)=$ $(-1)^{a_{11}(n)}$. It is a 2 -automatic sequence generated by an automaton of four states.

The paperfolding sequence $\mathbf{p}=p(0) p(1) p(2) \cdots$ is defined as follows: writing $(n)_{2} 00$ as $1^{i} 0 a w$ for some $i \geq 0$ some $a \in\{0,1\}$, and some $w \in\{0,1\}^{*}$, we have

$p(n)=(-1)^{a}$. It is a 2 -automatic sequence generated by an automaton of four states.

Theorem 5. The Rudin-Shapiro sequence has an unbordered factor of every length.

Proof. We applied the same technique discussed previously for the Thue-Morse sequence.

Here is a summary of the computation:

Stage 1: 269 states

Stage 2: 85313 states minimized to 1974

Stage 3: 48488 states minimized to 6465

Stage 4: 6234 states. 
The Stage 4 NFA has 6234 states. We were unable to determinize this automaton directly (using two different programs) due to an explosion in the number of states created. Instead, we reversed the NFA (creating an NFA for $L^{R}$ ) and determinized this instead. The resulting DFA has 30 states, and upon minimization, gives a 1-state automaton accepting all strings.

Theorem 6. The paperfolding sequence has an unbordered factor of length $n$ if and only if the reversed representation $(n)_{2}$ is rejected by the automaton given in Figure 4

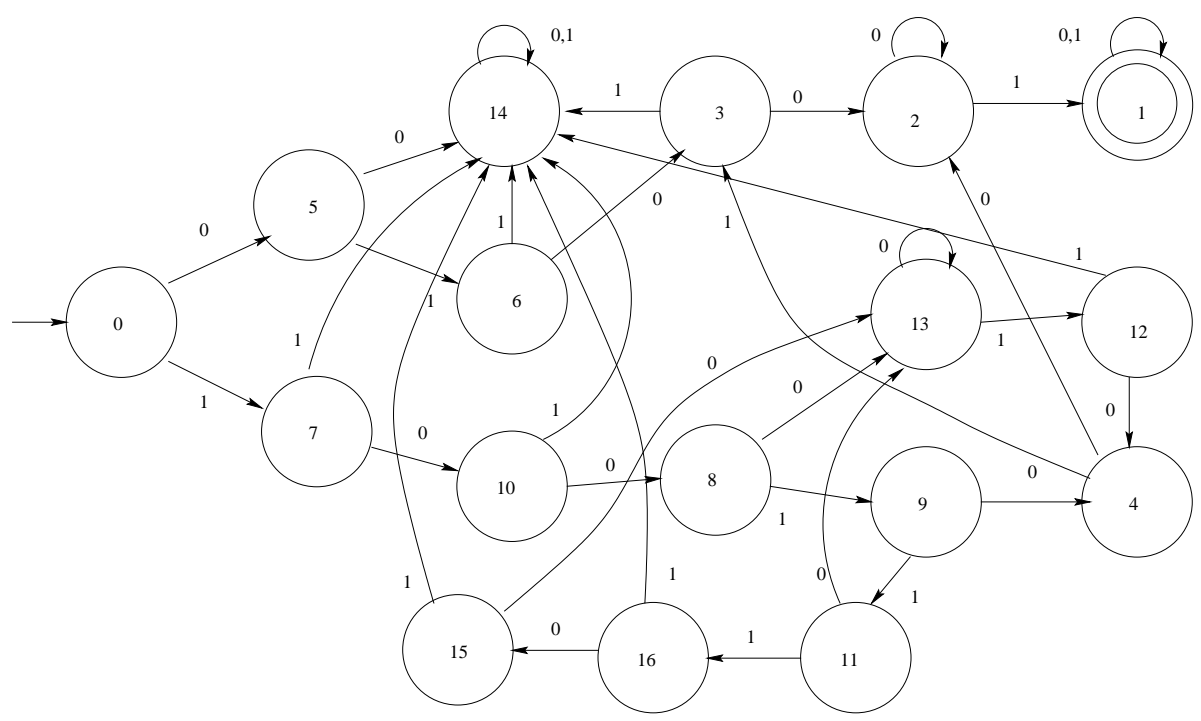

Fig. 2. A finite automaton for unbordered factors in the paperfolding word

Proof. We applied the same technique discussed previously for the Thue-Morse sequence.

Here is a summary of the computation: 6 seconds cpu time on a $2.9 \mathrm{GHz}$ Dell XPS laptop.

Stage 1, 159 states

Stage 2, 1751 minimized down to 89 states

Stage 3, 178 minimized down to 75 states

Stage 4, 132 minimize down to 17 states . 


\section{$5 \quad$ Further work}

In the future, we plan to extend this work to explicitly compute the number of distinct unbordered factors of length $n$ in the Thue-Morse sequence. (A conjecture about this number was given in $\underline{6}$.)

\section{Open problems}

Which of the problems mentioned in $\S 1$ are algorithmically decidable for the more general class of morphic sequences?

Can the techniques be applied to detect abelian powers in automatic sequences?

\section{References}

1. J.-P. Allouche, N. Rampersad, and J. Shallit. Periodicity, repetitions, and orbits of an automatic sequence. Theoret. Comput. Sci. 410 (2009), 2795-2803.

2. J.-P. Allouche and J. O. Shallit. The ubiquitous Prouhet-Thue-Morse sequence. In C. Ding, T. Helleseth, and H. Niederreiter, editors, Sequences and Their Applications, Proceedings of SETA '98, pp. 1-16. Springer-Verlag, 1999.

3. J.-P. Allouche and J. Shallit. Automatic Sequences: Theory, Applications, Generalizations. Cambridge University Press, 2003.

4. J. Berstel. Axel Thue's Papers on Repetitions in Words: a Translation. Number 20 in Publications du Laboratoire de Combinatoire et d'Informatique Mathématique. Université du Québec à Montréal, February 1995.

5. V. Bruyère, G. Hansel, C. Michaux, and R. Villemaire. Logic and p-recognizable sets of integers. Bull. Belgian Math. Soc. 1 (1994), 191-238. Corrigendum, Bull. Belg. Math. Soc. 1 (1994), 577.

6. E. Charlier, N. Rampersad, and J. Shallit. Enumeration and decidable properties of automatic sequences. In G. Mauri and A. Leporati, editors, Developments in Language Theory, 15th International Conference, DLT 2011, Vol. 6795 of Lecture Notes in Computer Science, pp. 165-179. Springer, 2011.

7. J. C. Costa. Biinfinite words with maximal recurrent unbordered factors. Theoret. Comput. Sci. 290 (2003), 2053-2061.

8. J. D. Currie and K. Saari. Least periods of factors of infinite words. RAIRO Inform. Théor. App. 43 (2009), 165-178.

9. F. M. Dekking, M. Mendès France, and A. J. van der Poorten. Folds! Math. Intelligencer 4 (1982), 130-138, 173-181, 190-195. Erratum, 5 (1983), 5.

10. J.-P. Duval. Une caractérisation de la période d'un mot fini par la longueur de ses facteurs primaires. C. R. Acad. Sci. Paris 290 (1980), A359-A361.

11. J.-P. Duval. Relationship between the period of a finite word and the length of its unbordered segments. Discrete Math. 40 (1982), 31-44.

12. J.-P. Duval, T. Harju, and D. Nowotka. Unbordered factors and Lyndon words. Discrete Math. 308 (2008), 2261-2264.

13. A. Ehrenfeucht and D. M. Silberger. Periodicity and unbordered segments of words. Discrete Math. 26 (1979), 101-109.

14. T. Harju and D. Nowotka. Periodicity and unbordered words: a proof of the extended duval conjecture. J. Assoc. Comput. Mach. 54 (2007), 1-20. 
15. S. Holub. A proof of the extended Duval's conjecture. Theoret. Comput. Sci. 339 (2005), 61-67.

16. S. Holub and D. Nowotka. On the relation between periodicity and unbordered factors of finite words. Internat. J. Found. Comp. Sci. 21 (2010), 633-645.

17. J. Honkala. A decision method for the recognizability of sets defined by number systems. RAIRO Inform. Théor. App. 20 (1986), 395-403.

18. P. T. Nielsen. A note on bifix-free sequences. IEEE Trans. Inform. Theory IT-19 (1973), 704-706.

19. N. Rampersad, J. Shallit, and M.-w. Wang. Inverse star, borders, and palstars. Inform. Process. Lett. 111 (2011), 420-422.

20. W. Rudin. Some theorems on Fourier coefficients. Proc. Amer. Math. Soc. 10 (1959), 855-859.

21. K. Saari. On the Frequency and Periodicity of Infinite Words. PhD thesis, University of Turku, Finland, 2008.

22. J. Shallit. The critical exponent is computable for automatic sequences. In P. Ambrož, S. Holub, and Z. Masáková, editors, WORDS 2011, 8th International Conference, pp. 231-239. Elect. Proc. Theor. Comput. Sci., 2011. Available at http://arxiv.org/abs/1104.2303v2.

23. H. S. Shapiro. Extremal problems for polynomials and power series. Master's thesis, MIT, 1952.

24. A. Thue. Über unendliche Zeichenreihen. Norske vid. Selsk. Skr. Mat. Nat. Kl. 7 (1906), 1-22. Reprinted in Selected Mathematical Papers of Axel Thue, T. Nagell, editor, Universitetsforlaget, Oslo, 1977, pp. 139-158.

25. A. Thue. Über die gegenseitige Lage gleicher Teile gewisser Zeichenreihen. Norske vid. Selsk. Skr. Mat. Nat. Kl. 1 (1912), 1-67. Reprinted in Selected Mathematical Papers of Axel Thue, T. Nagell, editor, Universitetsforlaget, Oslo, 1977, pp. 413478. 